\title{
Motherhood meets epidemiology: measuring risk factors for breast-feeding cessation
}

\author{
Jennifer K Peat ${ }^{1, *}$, Jane Allen ${ }^{2}$, Nguyen Nguyen $^{3}$, Andrew Hayen ${ }^{4}$, Wendy H Oddy ${ }^{5}$ \\ and Seema Mihrshahi ${ }^{1}$ \\ ${ }^{1}$ Clinical Epidemiology Unit, The Children's Hospital at Westmead, Locked Bag 4001, Westmead, New South Wales \\ 2145, Australia: 'James Fairfax Institute of Paediatric Nutrition, The Children's Hospital at Westmead, Westmead, \\ Australia: ${ }^{3}$ Garvan Institute of Medical Research, St Vincent Hospital, Sydney, Australia: ${ }^{4}$ NSW Department of \\ Health, Sydney, Australia: ${ }^{5}$ School of Public Health, Curtin University of Technology, Telethon Institute for Child Health \\ Research, Perth, Australia
}

Submitted 5 January 2004: Accepted 19 May 2004

\begin{abstract}
Objective: To examine statistical models that have been used to predict the cessation of breast-feeding.

Setting: In nutritional epidemiology, a knowledge of risk factors that lead to breastfeeding cessation is essential to promote optimal infant health by increasing or sustaining breast-feeding rates. However, a number of methodological issues complicate the measurement of such risk factors. It is important when building multivariate models that variables entered into the model are not intervening variables, factors on the causal pathway or surrogate outcomes. Inclusion of these types of variable can lead to inaccurate models and biased results. A factor often cited to predict breast-feeding is 'intention to breast-feed' prior to the birth of the infant, although this factor is directly on the causal decision-making pathway. Another factor often cited is the age of introduction of formula feeding, which is actually part of the outcome variable because formula feeding defines the difference between full, complementary and no breast-feeding. Rather than include these as risk factors in multivariate models, factors removed from the causal pathway such as influences of educational practices, including advice to complementary feed, and beliefs and attitudes of families and health-care practitioners should be measured.

Conclusions: The accurate quantification of modifiable risk factors is essential for designing public health education campaigns that are effective in sustaining or increasing breast-feeding duration.
\end{abstract}

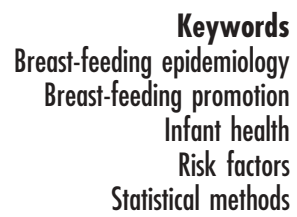

In this paper, we use the following definitions:

- Full breast-feeding to describe either exclusive breastfeeding, i.e. infants who receive breast milk as their only source of nutrition except for vitamin/mineral supplements or medicines, or predominant breast-feeding, i.e. infants who are breast-fed and receive liquids (water and water-based drinks, fruit juice, oral rehydration solution), ritual fluids and vitamin/mineral supplements or medicines, but who do not receive infant formula or solid or semi-solid foods.

- Complementary breast-feeding to describe infants who receive breast milk but who also receive infant formula or solid or semi-solid foods.

- No breast-feeding to describe infants who receive no breast milk.

A large body of evidence for risk factors that are associated with the initiation and duration of breast-feeding has been reviewed $^{1}$. However, a number of key issues complicate the interpretation of risk factor evidence. One problem that sometimes arises is that methodologically sound approaches to building multivariate statistical models are not always used. An issue that is frequently overlooked is that any variable considered in a model must be a potentially predictive factor but must not be an intervening variable, a factor on the causal pathway or a surrogate outcome $e^{2,3}$. The inclusion of these types of variable in multivariate models can bias measures of effect and mask the importance of true risk factors.

Restricting the use of infant formula feeding has been one of the most cost-effective health interventions identified $^{4}$. However, while current breast-feeding rates remain below health targets, it is clear that new approaches to further increase breast-feeding rates are needed. Health promotions to increase breast-feeding duration need to be based on sound evidence of risk factors that predict whether mothers decline to initiate breast-feeding or stop breast-feeding at an early stage. 
To obtain this information, epidemiological studies that adhere to good practice and that use established methodological and statistical principles are essential.

In this paper, we consider the methods used to generate risk factor information for breast-feeding duration and discuss alternative methods that are required for informing effective public health campaigns to promote an increase in breast-feeding duration in the population.

\section{Background}

The evidence that breast-feeding, particularly exclusive breast-feeding, has a wide range of important health benefits for both infants and mothers ${ }^{5,6}$ is supported by knowledge of multiple biochemical pathways that confer anti-bacterial, anti-fungal and antigenic effects ${ }^{7,8}$. For these reasons, a number of health authorities now recommend exclusive breast-feeding for the first 6 months of life and complementary breast-feeding for 1 to 2 years $^{9-11}$. Although there has been a gradual increase in rates of breast-feeding in developed countries since the $1980 s^{12,13}$, a minority of infants are exclusively breast-fed for the first 6 months. National survey data in Australia suggests that, at 3 months, only 57\% of infants are fully breast-fed and at 6 months, only 19\% are fully breast-fed and $53 \%$ receive no breast milk after this time ${ }^{14}$. These rates may vary between surveys because of the methods used, but all rates remain well below national and international health targets.

In developing countries, the protective effect of breastfeeding is essential for child health through its role in preventing major causes of child mortality including gastrointestinal and respiratory infections. During the 1990s, modest improvements were made in exclusive breast-feeding for the first 4 months of life, with rates increasing from 48 to $52 \%$ in the developing world based on 37 countries with trend data. Timely complementary feedings at 6-9 months have also improved, with rates increasing from 43 to $49 \%$ between 1990 and $2000^{15}$. In developing countries, the protection afforded by breastfeeding increases with duration and exclusivity and continues into the second year of life, although the risk is reduced over time $e^{5}$. In countries or communities with high child mortality, it is especially important to increase the maintenance of breast-feeding practices.

\section{Causal pathways and surrogate outcomes}

Good epidemiological practice in measuring risk factors for breast-feeding cessation is essential. In this, risk factors that are identified should be potential predictors of breastfeeding practices but must not be on the causal pathway. If a considered factor is on the causal pathway to the initiation of breast-feeding, it must not be included in a multivariate model.

A factor often used to predict breast-feeding is 'intention to breast-feed ${ }^{16-19}$. Intention to change is the basis of health promotion theory, where it is clearly an interim step on the pathway to altered behaviour. Figure 1 shows how 'intention' is directly on the causal decision-making pathway to breast or complementary feeding patterns. As with any behaviour, it is unlikely that someone would engage in breast-feeding without the intention to do so. With acknowledged factors such as this, we need to investigate the influences that lead to the decision and not treat the decision itself as a risk factor. We also need to acknowledge that there may be intervening variables such as cracked nipples or other breast-feeding problems that may lead to breast-feeding cessation.

In addition to excluding causal pathway factors and intervening variables, surrogate outcomes must be treated carefully. Because they are associated with the outcome, surrogate outcomes inevitably distort multivariate models. From the definitions of breast-feeding, it is clear that formula feeding is part of the outcome variable because it is the alternative feeding method. Although the introduction of formula actually defines the difference between full and complementary or no breast-feeding, early introduction of formula is often cited as a risk factor for breastfeeding cessation ${ }^{16,20-22}$. Planning to introduce formula feeding is also intuitively on the decision-making pathway. This inherently strong association between ceasing to breast-feed and introduction of formula feeds precludes its consideration as a risk factor.

\section{Influential factors}

The introduction of infant formula has been described as a symptom rather than a cause of breast-feeding difficulties $^{17}$. The extent to which complementary feeding interferes with infants' feeding behaviours and undermines

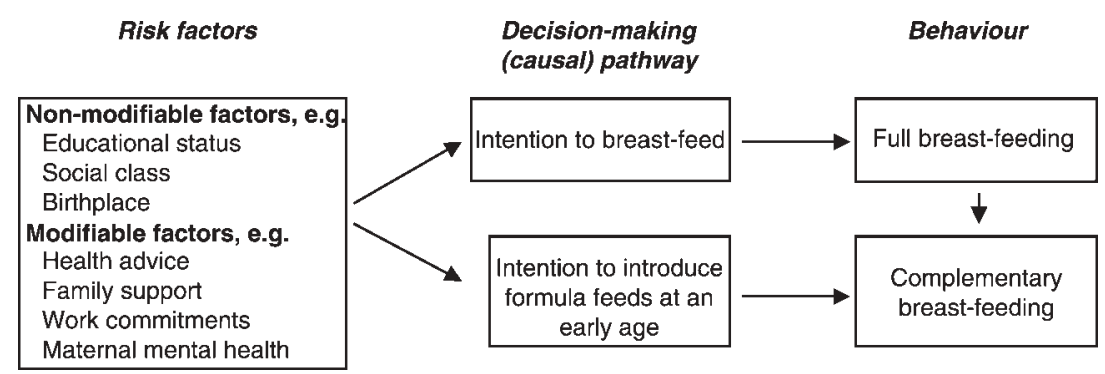

Fig. 1 Causal/decision-making pathway to uptake and duration of breast-feeding 
mothers' confidence may be driven by infants who are difficult feeders or may be a marker of poor advice from health practitioners ${ }^{4}$. Instead of viewing the introduction of formula as a risk factor for breast-feeding cessation, the reasons behind the introduction, which are the real risk factors, must be measured accurately.

Influences that lead to infant feeding decisions may include advice to complementary feed from health-care practitioners. Measures of the effects of advice have much greater practical application as modifiable risk factors than measuring the age of the introduction of formula. Other risk factors include subtle marketing techniques targeted at paediatricians and general practitioners by infant formula companies ${ }^{23}$. Although marketing influences are difficult to quantify, inclusion of the receipt of advice or awareness of marketing messages to complementary feed in a multivariate model would be sound in that these factors are removed from the causal pathway. The importance of measuring reasons for breast-feeding decisions and the cultural beliefs and traditions underlying breast-feeding decisions is recognised ${ }^{24}$.

\section{Epidemiological issues}

There are other epidemiological issues in measuring risk factors for breast-feeding that have been reviewed ${ }^{1,19,25}$. In observational studies, factors such as selection bias, confounding and reverse causality need to be considered $^{24}$. For example, research participants may not be randomly sampled so that the results are not generalisable to the entire population, and the types of risk factor included in models vary widely so that comparability across studies is compromised. Other issues include low statistical power, measurement error and bias arising from misclassification, selection, recall and reporting of information $^{26}$.

The collection of risk factor information is not a simple task, but assessing the effect of advice and other factors on the decision to introduce formula feeding is a research area in need of urgent attention. In practice, both outcome and risk factor information are usually collected by interview or questionnaire, which is generally easy to obtain, but reporting bias will always be a major issue when mothers are aware of the benefits of breast-feeding. In future studies, influential risk factor information may be more difficult to quantify accurately because of its subtlety and a greater potential for reporting and recall bias. For example, it may be important to measure the impact of formula advertising in parenting magazines or of advice to complementary feed from a health professional. In this, well-designed cohort or experimental studies are essential.

In any study, the reporting of unadjusted associations between breast-feeding and risk factors may be misleading. For accurate information, it is important that multivariate statistical analyses are used to measure the adjusted, i.e. independent, effects. Large longitudinal cohort studies could provide the statistical power needed to support such complex analyses, but the costs and skills required to implement such studies can be prohibitive. Nevertheless, another generation of breast-feeding epidemiology with a fresh approach to informing public health campaigns is needed. This could begin with a re-analysis of existing data using more stringent methods, but will also require new, more rigorous research to collect a high level of evidence.

The methods used to analyse risk factor data will depend to a large extent on the study design. For cohort studies, survival analyses provide the most useful tool for investigating factors that influence breast-feeding duration because they make the most value of data from mothers who are still breast-feeding and factors such as time of follow-up. In cross-sectional studies, analyses are usually limited to multiple and logistic regression with no period effect so that the measurement of factors that influence rates of change over time is precluded.

\section{Non-modifiable and modifiable risk factors}

The development of effective breast-feeding public health promotion campaigns centres on addressing modifiable risk factors after taking non-modifiable risk factors into account. Non-modifiable risk factors for breast-feeding include mother and infant characteristics such as maternal age, ethnicity, social class, having a partner, educational attainment, gestation age, parity, gender and, in some situations, mode of delivery of the baby. These nonmodifiable factors can be used to identify and target groups who are at high risk of deciding to introduce formula feeds.

In countries where beliefs about breast-feeding are influenced by local culture ${ }^{27}$, targeted interventions will be especially important. Interpretation of the results of studies in developing countries is difficult because of many confounding factors such as the child's health at time of weaning in addition to cultural habits and taboos. In such countries, a higher social class implies increased affordability of infant formula and may be on the causal pathway to stopping breast-feeding.

For designing any public health intervention, an accurate and locally applicable knowledge of modifiable risk factors is essential. The many modifiable risk factors for breast-feeding duration include paternal and family support, smoking, need to return to work and the nature of the working environment ${ }^{1}$. Other important modifiable factors may include attitudes of health-care workers and family members, access to nutritional advice, psychosocial factors such as mental health of the mother including maternal stress, depression and coping mechanisms, and the effects of marketing campaigns. Factors such as the time that infant feeding decisions are made $^{28}$, which is influenced by previous experiences in addition to the 
factors above, are important for maximising the effectiveness of health promotions by planning interventions at critical periods.

\section{Recommendations}

To increase breast-feeding rates further, it is important that a high level of information about the influences of educational practices, such as advice to complementary feed, is collected. Although experimental approaches to measuring influences on breast-feeding have generally been precluded, the effectiveness of interventions to initiate and maintain breast-feeding ${ }^{29}$ and the effects of planned introductions of formula feeding ${ }^{17}$ have been measured in randomised controlled trials. Collecting a high level of evidence of the effects of health messages about complementary feeding could be achieved in randomised trials, including cluster-designed trials to prevent contamination between study groups. Such trials will need to address many issues, including the difficulties in implementing changes in hospital and health professional practices, problems of staff attitudes to new routines and the need for skilled help to prevent or overcome breast-feeding difficulties ${ }^{30}$.

Accuracy in measuring risk factors for shorter breastfeeding duration has the potential to lead to more effective health campaigns and better educational messages. In the future, the effects of feeding recommendations and of the beliefs and attitudes of both parents and health-care workers will be estimated more accurately if factors on the causal pathway and surrogate outcomes are excluded from multivariate models. This model building approach is essential for collecting accurate information in all health disciplines. In promoting healthy infant feeding, the development of effective campaigns that are locally tailored for clearly identified groups of mothers, delivered at critical time points and built on rigorous models will maximise our potential to sustain or increase rates of breast-feeding in both developed and developing countries.

\section{References}

1 Scott JA, Binns CW. Factors associated with the initiation and duration of breastfeeding: a review of the literature. Australian Journal of Nutrition and Dietetics 1998; 55 : 51-61.

2 Rothman KJ. Modern Epidemiology. Boston, MA: Little, Brown and Company, 1986; 125-30.

3 Hennekens $\mathrm{CH}$, Buring JE. Analysis of epidemiologic studies: evaluating the role of confounding. In: Mayrent SL, ed. Epidemiology in Medicine. Boston, MA: Little, Brown and Company, 1987; 287-321.

4 World Health Organization (WHO). Evidence for the Ten Steps to Successful Breastfeeding. WHO/CHD/98.9. Geneva: WHO, 1998.

5 WHO Collaborative Study Team on the Role of Breastfeeding on the Prevention of Infant Mortality. Effect of breastfeeding on infant and child mortality due to infectious diseases in less developed countries. Lancet 2000; 355 $451-5$.

6 Leon-Cava N, Lutter C, Ross J, Martin L. Quantifying the Benefits of Breastfeeding: A Summary of the Evidence. The Linkages Project. Washington, DC: Pan American Health Organization, 2002.

7 Brandtzaeg P. Mucosal immunity integration between mother and the breast-fed infant. Vaccine 2003; 21: 3382-8.

8 Oddy W. Breastfeeding protects against illness and infection in infants and children: a review of the evidence. Breastfeeding Review 2001; 9: 11-8.

9 American Academy of Pediatrics. Breastfeeding and the use of human milk. Pediatrics 1997; 100: 1035-9.

10 Michaelsen KF, Weaver L, Branca F, Robertson A. Feeding and Nutrition of Infants and Young Children: Guidelines for the WHO European Region, with Emphasis on the Former Soviet Countries. Copenhagen: World Health Organization Regional Office for Europe, 2000.

11 World Health Organization (WHO). The Optimal Duration of Exclusive Breastfeeding. Geneva: WHO, 2001.

12 National Health and Medical Research Council. Dietary Guidelines for Children and Adolescents in Australia. Canberra: Commonwealth of Australia, 2003.

13 Wright AL. The rise of breastfeeding in the United States. Pediatric Clinics of North America 2001; 48: 1-12.

14 Donath S, Amir LH. Rates of breastfeeding in Australia by state and socio-economic status: evidence from the 1995 National Health Survey. Journal of Paediatric and Child Health 2000; 36: 164-8.

15 http://www.childinfo.org/eddb/brfeed/test/database.htm

16 Loughlin HH, Clapp-Channing NE, Gehlbach SH, Pollard JC, McCutchen TM. Early termination of breast feeding; indentifying those at risk. Pediatrics 1985; 75: 508-13.

17 Cronenwett L, Stukel T, Kearney M, Barrett J, Covington A, Del Monte K, et al. Single daily bottle use in the early weeks postpartum and breast feeding outcomes. Pediatrics 1992; 90: 760-6.

18 Lawson K, Tulloch MI. Breast feeding duration: prenatal intentions and postnatal practices. Journal of Advanced Nursing 1995; 22: 841-9.

19 Quarles A, Williams PD, Hoyle DA, Brimeyer M, Williams AR. Mothers' intention, age, education and the duration and management of breast feeding. Maternal-Child Nursing Journal 1994; 22: 102-8.

20 Hornell A, Hofvander Y, Kylberg E. Solids and formula: association with pattern and duration of breastfeeding. Pediatrics 2001; 107: 31-7.

21 Dulon M, Kersting M, Schach S. Duration of breastfeeding and associated factors in Western and Eastern Germany. Acta Paediatrica 2001; 90: 931-5.

22 Hall RT, Mercer AM, Teasley SL, McPherson DM, Simon SD, Santos SR, et al. A breastfeeding assessment score to evaluate the risk for cessation of breastfeeding by 7 to 10 days of age. Journal of Pediatrics 2002; 141: 659-64.

23 Henderson L, Kitzinger J, Green J. Representing infant feeding: content analysis of British media portrayals of bottle feeding and breast feeding. British Medical Journal 2000; 321: 1196-8.

24 Kramer K. Breastfeeding and child health, growth, and survival [Commentary]. International Journal of Epidemiology 2003; 32: 96-8.

25 Rutishauser IHE, Carlin JB. Body mass index and duration of breastfeeding: a survival analysis during the first six months of life. Journal of Epidemiology and Community Health 1992; 46: 559-65.

26 Oddy WH, Peat JK. Breastfeeding, asthma and atopic disease: an epidemiological review of the literature. Journal of Human Lactation 2003; 19: 250-61.

27 Diong S, Johnson M, Langdon R. Breastfeeding and Chinese 
mothers living in Australia. Breastfeeding Review 2000; 8: $17-23$.

28 Scott JA, Landers MCG, Hughes RM, Binns CW. Factors associated with breastfeeding at discharge and duration of breastfeeding. Journal of Paediatrics and Child Health 2001; 37: 254-61.

29 Kramer MS, Chalmers B, Hodnett ED, Sevkovskaya Z,
Dzikovich I, Shapiro S, et al. Promotion of breastfeeding intervention trial (PROBIT). A randomized trial in the Republic of Belarus. Journal of the American Medical Association 2001; 285: 413-20.

30 Kramer MS, Kakuma R. Optimal duration of exclusive breastfeeding. Cochrane Database of Systematic Reviews 2002: CD003517. 